\title{
Cambios metodológicos para la adquisición y evaluación de la competencia transversal "Conocimiento de los problemas contemporáneos"
}

A. Bes-Piá, J.A. Mendoza-Roca, A. Iborra-Clar, E. Ferrer-Polonio, E. Zuriaga-Agustí Departamento de Ingeniería Química y Nuclear. Universitat Politècnica de València. mbespia@iqn.upv.es; $\quad$ jamendoz@iqn.upv.es; aiborra@iqn.upv.es; $\quad$ evferpo@posgrado.upv.es elzuag@etsii.upv.es

\begin{abstract}
This article refers to the changes introduced in the methodology of a master's subject for the acquisition and evaluation of the transversal competence "Knowledge of contemporary problems" (CT-10 of the UPV). The need for introducing changes in the previous methodology arises from the evaluation carried out by the subject lecturers, when detecting that the levels of domain I (identification) and II (analysis) contemplated in the rubric of evaluation were not consolidated at the level of master, corresponding to domain III (proposal of solutions and evaluation). The methodological changes introduced are herewith justified. This work aims to be an example of teaching experience for the acquisition of this key competence. It has been revealed as a significant aspect for the students, the achievement of the best solutions for problems or challenges in any professional field, through the identification, analysis and evaluation of the proposed alternatives.
\end{abstract}

Keywords: transversal competence, contemporary problems, competence evaluation, environment

\begin{abstract}
Resumen
En este trabajo se explican los cambios metodológicos introducidos, en una asignatura de máster, para la adquisición y evaluación de la competencia transversal "Conocimiento de problemas contemporáneos" (CT-10) de la UPV. La necesidad de realizar cambios en la metodología inical, que se definió en su momento, surge tras la evaluación realizada por el profesorado de la asignatura al detectar que los niveles de dominio I y II, contemplados en la rúbrica de evaluación de dicha competencia, no estaban consolidados a nivel de máster, correspondiente al nivel de dominio III. Los cambios metodológicos introducidos se justifican en cada etapa establecida. El trabajo pretende ser un ejemplo de experiencia docente para la adquisición de una competencia tan fundamental como es el conocimiento y búsqueda de la mejor solución, ante los problemas o retos que puedan surgir en cualquier campo profesional, a través de la identificación, análisis y evaluación de las soluciones propuestas.
\end{abstract}

Palabras clave: competencia transversal, problemas contemporáneos, evaluación competencia, medio ambiente 
Cambios metodológicos para la adquisición y evaluación de la competencia transversal "Conocimiento de los problemas contemporáneos"

\section{Introducción}

El proyecto sobre "Competencias Transversales" (CT) de la Universitat Politècnica de València (UPV) es un proyecto ambicioso que tiene como objetivo principal acreditar que los alumnos egresados, de cualquier titulación impartida en la UPV, hayan adquirido un total de trece competencias (UPV, 2018). Estas competencias son transversales porque pueden ser transferibles entre contextos personales, sociales, académicos y laborales a lo largo de la vida, por lo que son clave para favorecer la inserción laboral de los estudiantes. Para asegurar su adquisición, cada competencia se debe trabajar y evaluar de forma continua en diferentes niveles o "dominios" competenciales en cada una de las titulaciones (Dominio I: $1^{\circ}$ y $2^{\circ}$ Grado; Dominio II: $3^{\circ}$ y $4^{\circ}$ Grado; Dominio III: Máster).

Una de las vías para la incorporación de las CTs consiste en asignarlas a diferentes asignaturas de cada titulación convirtiéndose en asignaturas "punto de control". En dichas asignaturas los profesores deben diseñar actividades y recoger evidencias que evalúen la adquisición de las CTs por parte de los estudiantes. Por ello, los profesores debemos ir adaptando nuestras metodologías docentes para que los estudiantes adquieran de forma conjunta tanto los conocimientos propios de la asignatura como el "saber hacer complejo" que implican las competencias transversales. En esta tarea no se debe olvidar que el eje de acción principal son los estudiantes así como su aprendizaje desde el punto de vista conceptual, procedimental y actitudinal.

Existen varias publicaciones donde se aborda el cambio metodológico para el aprendizaje de las competencias. De éstas cabe destacar una donde se indica la relación existente entre los métodos de enseñanza y las competencias (De Miguel, 2006), y otra, en el que se establecen los criterios para la enseñanza de las competencias. Entre los criterios que se indican cabe destacar el de la "enseñanza significativa" como elemento clave para el aprendizaje (Zabala, 2008). A esto cabe añadir que el interés generado por parte del profesorado en la adquisición y evaluación de competencias dentro de sus materias hace que la innovación en competencias sea una línea prioritaria en los proyectos de innovación docente (Quirós et al, 2018).

Una de las metodologías docentes empleadas para la adquisición de CTs es el debate. Hay varias experiencias del uso de esta herramienta cuyo uso es evaluado positivamente (Ortega, 2017) (Barrenetxea, 2018). En este trabajo se incluye el uso de esta metodología dado que favorece la adquisición de otras competencias como es el desarrollo de habilidades comunicativas.

Otro aspecto importante a tener en cuenta es la evaluación que se realiza de las competencias (Fernández, 2018). Las referencias sobre trabajos encontrados se centran mayoritariamente en el uso de las rúbricas como herramienta de evaluación de competencias tanto transversales como específicas (Herrero, 2014) (Mudarra, 2012) (Piqué, 2012). En este trabajo, se emplea la rúbrica como instrumento clave para la evaluación de la competencia "Conocimiento de los problemas contemporáneos" (CT-10). 


\section{Objetivos}

El principal objetivo del trabajo es modificar la metodología docente, utilizada en una asignatura de máster, para la adquisición y evaluación de la competencia "Conocimiento de los problemas contemporáneos". Mediante el cambio metodológico se pretende trabajar y consolidar los tres niveles de dominio de esta competencia (I y II Grado y III Máster) así como detectar carencias que puedan encontrarse debido a la todavía reciente implantación del proyecto de competencias transversales de la UPV.

\section{Desarrollo de la innovación}

\subsection{Contextualización de la asignatura}

Se trata de una asignatura de la materia "bioprocesos aplicados al medio ambiente", que es una materia optativa del Máster en Ingeniería Química, impartida durante el primer semestre de segundo curso. Es una asignatura muy aplicada que pone en contacto a los alumnos con su campo profesional no sólo a través de los conocimientos teóricos sino a través de las prácticas de laboratorio y visitas de campo ( 6 créditos ECTS). Esta asignatura es "punto de control" para la competencia "Conocimiento de los problemas contemporáneos". El número de alumnos es reducido (10-15) lo que permite realizar innovaciones docentes así como una evaluación de la adquisición de la competencia de forma continuada a los estudiantes. Mayoritariamente, se trata de estudiantes con un perfil con un nivel formativo amplio y con una alta motivación.

\subsection{Antecedentes. Metodología previa (M_1)}

La idea surge después de evaluar durante tres cursos académicos consecutivos, entre el profesorado de la asignatura, la metodología utilizada para la adquisición y evaluación de la competencia "Conocimiento de los problemas contemporáneos". Durante estos tres cursos, se establecieron como indicadores de referencia la rúbrica guía proporcionada por el Instituto de las Ciencias de la Educación, en concreto, los correspondientes a un nivel de dominio III, dado que la asignatura es de máster. Los indicadores de este nivel sugieren "proponer soluciones a los problemas de un campo concreto profesional". Por ello, el primer diseño metodológico realizado (M_1) para trabajar dicha competencia consistió en proponer por parte del profesorado un problema en el campo profesional del medio ambiente y que los alumnos aportaran soluciones desde el punto de vista técnico y medioambiental. Para ello, la metodología se dividió en tres fases: la primera fase consistía en el planteamiento del problema por parte de los profesores apoyado con la visualización de dos videos (25 minutos). En la segunda fase de la actividad, los alumnos debían proponer soluciones trabajando en grupo de 3-4 personas. Para esta parte, los alumnos disponen de un documento "review" sobre el problema planteado en el ámbito de la Unión Europea y que deben consultar. Además, se entrega un cuestionario con 4 preguntas concretas que deben contestar y utilizar para el debate posterior (fase 3), que se establece entre los grupos de trabajo, con la finalidad de buscar la mejor solución entre todos en base a criterios establecidos. Cabe indicar que toda la actividad se desarrollaba en una sesión de aula de $2 \mathrm{~h}$. Las evidencias para evaluar la adquisición de la competencia se centraban en la respuesta a las preguntas de forma individual por parte de cada alumno. 
Cambios metodológicos para la adquisición y evaluación de la competencia transversal "Conocimiento de los problemas contemporáneos"

En la Tabla 1 se recogen los aspectos positivos y negativos en cada una de las partes de la metodología inicial (M_1), fruto de la reflexión docente.

Tabla 1. Propuesta metodológica y evaluación de fases para la CT-10 (M_1)

\begin{tabular}{|c|c|c|c|}
\hline Fase actividad & $\begin{array}{c}\text { Metodología } \\
\text { docente } \\
\text { (duración) }\end{array}$ & $\begin{array}{l}\text { Recursos } \\
\text { didácticos }\end{array}$ & $\begin{array}{c}\text { Aspectos positivos/negativos } \\
\text { de la fase }\end{array}$ \\
\hline $\begin{array}{l}\text { FASE 1: } \\
\text { Introducción tarea } \\
\text { - } \quad \text { Objetivo actividad } \\
\text { - } \quad \text { Exposición problema } \\
\text { - } \quad \text { Modo evaluación }\end{array}$ & $\begin{array}{l}\text { Lección } \\
\text { magistral } \\
(20 \mathrm{~min})\end{array}$ & $\begin{array}{l}\text { Videos } \\
\text { Rúbrica }\end{array}$ & $\begin{array}{l}\checkmark \text { Definición objetivo y modo } \\
\text { evaluación } \\
\times \text { Los alumnos parten de un } \\
\text { problema definido pero no se } \\
\text { favorece la reflexión crítica }\end{array}$ \\
\hline $\begin{array}{l}\text { FASE 2: } \\
\text { Propuesta de soluciones } \\
\text { - } \quad \text { Lectura documento } \\
\text { - } \quad \text { Elaboración propuestas } \\
\text { - } \quad \text { Contraste de opiniones } \\
\quad \text { intergrupal }\end{array}$ & $\begin{array}{l}\text { Trabajo } \\
\text { grupal } \\
3-4 \text { personal } \\
(60 \mathrm{~min})\end{array}$ & $\begin{array}{l}\text { Documento } \\
\text { "review" } \\
\text { Cuestionario }\end{array}$ & $\begin{array}{l}\sqrt{ } \text { Trabajo grupal, intercambio ideas } \\
\times \text { Documento "review" extenso, una } \\
\text { fuente de consulta. } \\
\times \text { Fuente de información limitada y } \\
\text { sin posibilidad de poder contrastar } \\
\text { otras fuentes. Las soluciones se basan } \\
\text { en una fuente de información. } \\
\text { × La propuesta de soluciones no } \\
\text { implica la identificación y análisis } \\
\text { previo (causas y consecuencias) del } \\
\text { problema }\end{array}$ \\
\hline $\begin{array}{l}\text { FASE 3: } \\
\text { Debate } \\
\text { - } \quad \begin{array}{l}\text { Exposición de } \\
\text { propuestas }\end{array} \\
\text { - } \quad \begin{array}{l}\text { Debate pro/contra de } \\
\text { soluciones }\end{array}\end{array}$ & $\begin{array}{l}\text { Debate } \\
(40 \mathrm{~min})\end{array}$ & Cuestionario & $\begin{array}{l}\checkmark \text { Fomento habilidades } \\
\text { comunicativas } \\
\checkmark \text { Fomento reflexión crítica } \\
\checkmark \text { Fomento participación activa } \\
\times \text { Criterios establecidos limitan el } \\
\text { debate. } \\
\times \text { El cuestionario limita las } \\
\text { respuestas. } \\
\times \text { Trabajo grupal pero cuestionario es } \\
\text { individual. } \\
\times \text { Se echa en falta "feed-back" de la } \\
\text { actividad y autoevaluación de los } \\
\text { estudiantes. }\end{array}$ \\
\hline
\end{tabular}


La evaluación de la adquisición de la competencia mediante la rúbrica indicó que:

- El 75\% de los estudiantes respondió de forma satisfactoria a las preguntas, mientras que sólo un $25 \%$ obtuvo la máxima calificación en la rúbrica.

- Las soluciones aportadas a través del cuestionario y contrastadas en el debate indican que los niveles de dominio I (identificación problema) y II (análisis problema: causas/consecuencias) no han sido trabajados por los alumnos. Esto se debe a que en la FASE 1 ya se plantea el problema como tal, sin dar cabida a la reflexión y análisis (dominio I). Por otro lado, tampoco se realiza un análisis del problema donde se debería analizar qué causa el problema y cuáles son las consecuencias (dominio II).

Cabe indicar, que la implantación de las CTs está todavía en proceso y que, ésta en concreto, es difícil de adaptar sobre todo en los estudios de grado, donde se trabaja el nivel de dominio competencial I y II.

\subsection{Metodología a implantar (M_2)}

A partir de las reflexiones recogidas en la Tabla 1, se plantea el cambio en la metodología que incluye la parte de identificación y análisis del problema. De esta manera, nos podremos asegurar una adecuada consolidación de la competencia en los tres niveles. En la Tabla 2, se muestran los cambios introducidos en la metodología previa (M_1). 
Cambios metodológicos para la adquisición y evaluación de la competencia transversal "Conocimiento de los problemas contemporáneos"

Tabla 2. Cambios introducidos en la metodología

\begin{tabular}{|c|c|c|c|}
\hline $\begin{array}{c}\text { Aspecto negativo } \\
\text { detectado }\end{array}$ & Propuesta de mejora & $\begin{array}{l}\text { Nuevos } \\
\text { indicadores } \\
\text { rúbrica } \\
\text { evaluación }\end{array}$ & Nuevo recurso \\
\hline $\begin{array}{l}\times \text { Los alumnos parten } \\
\text { de un problema } \\
\text { definido pero no se } \\
\text { favorece la reflexión } \\
\text { crítica. }\end{array}$ & $\begin{array}{l}\text { Los estudiantes deben } \\
\text { identificar porqué es un } \\
\text { problema. }\end{array}$ & $\begin{array}{l}\text { Se incluye en } \\
\text { la rúbrica. } \\
\text { Dominio I: } \\
\text { identificación }\end{array}$ & \multirow{3}{*}{$\begin{array}{l}\text { Cada grupo debe } \\
\text { elaborar un informe } \\
\text { breve con la } \\
\text { identificación y análisis. } \\
\text { Este será el punto de } \\
\text { partida para pasar al } \\
\text { nivel de dominio III. }\end{array}$} \\
\hline $\begin{array}{l}\text { × Documento "review" } \\
\text { extenso, una fuente de } \\
\text { consulta. } \\
\text { × Fuente de } \\
\text { información limitada y } \\
\text { sin posibilidad de poder } \\
\text { contrastar otras fuentes. } \\
\text { Las soluciones se basan } \\
\text { en una fuente de } \\
\text { información. }\end{array}$ & $\begin{array}{l}\text { Se aporta el documento } \\
\text { "review" como una fuente } \\
\text { posible de consulta. } \\
\text { Se valorará la búsqueda de } \\
\text { información de diferentes } \\
\text { fuentes: noticias, libros, } \\
\text { documentales, etc. } \\
\text { relacionado con el tema para } \\
\text { contrastar diferentes puntos } \\
\text { de vista del problema }\end{array}$ & $\begin{array}{l}\text { Se incluye en } \\
\text { la rúbrica. } \\
\text { Dominio I: } \\
\text { identificación }\end{array}$ & \\
\hline $\begin{array}{l}\text { × La propuesta de } \\
\text { soluciones implica la } \\
\text { identificación y análisis } \\
\text { previo (causas y } \\
\text { consecuencias) del } \\
\text { problema }\end{array}$ & $\begin{array}{l}\text { Los alumnos deben identificar } \\
\text { de forma breve y concisa las } \\
\text { causas y consecuencias sobre } \\
\text { el problema propuesto. }\end{array}$ & $\begin{array}{l}\text { Se incluye en } \\
\text { la rúbrica. } \\
\text { Dominio II: } \\
\text { análisis }\end{array}$ & \\
\hline $\begin{array}{l}\times \text { Criterios establecidos } \\
\text { limitan el debate. } \\
\times \text { El cuestionario limita } \\
\text { las respuestas. } \\
\times \text { Trabajo grupal pero } \\
\text { cuestionario es } \\
\text { individual. }\end{array}$ & $\begin{array}{l}\text { La búsqueda de soluciones al } \\
\text { problema no se acota con } \\
\text { criterios. } \\
\text { Se sustituye el cuestionario } \\
\text { por una propuesta de } \\
\text { soluciones por el grupo, no } \\
\text { individual. }\end{array}$ & $\begin{array}{l}\text { Se incluye en } \\
\text { la rúbrica. } \\
\text { Dominio III: } \\
\text { propuesta y } \\
\text { evaluación de } \\
\text { soluciones }\end{array}$ & $\begin{array}{l}\text { Redactar propuesta de } \\
\text { soluciones con los pros } \\
\text { y contras }\end{array}$ \\
\hline $\begin{array}{l}\text { × Se echa en falta } \\
\text { "feed-back" de la } \\
\text { actividad y } \\
\text { autoevaluación de los } \\
\text { estudiantes. }\end{array}$ & $\begin{array}{l}\text { Los alumnos se autoevalúan } \\
\text { con la rúbrica. } \\
\text { Realizan una valoración de la } \\
\text { actividad a través de una } \\
\text { encuesta. }\end{array}$ & & $\begin{array}{l}\text { Rúbrica } \\
\text { Encuesta valoración } \\
\text { actividad }\end{array}$ \\
\hline
\end{tabular}

A partir de los cambios propuestos en la Tabla 2 se establece la nueva propuesta metodológica la CT-10 (M_2) (Tabla 3). En primer lugar, cabe comentar que, para poder llevar a cabo estos cambios se requiere más tiempo. Por ello, en la propuesta se ha 
duplicado el tiempo global necesario, hasta 4 horas, para llevar a cabo esta actividad. Con el objetivo de no sobresaturar al alumno con actividades fuera del aula, se propone trabajar siempre en horario lectivo de la asignatura. De esta forma nos aseguramos que trabajan de forma grupal, además de poder consultar directamente a los profesores que tendrán una función de guía. Como se puede ver en la Tabla 3, se establecen 4 fases en la actividad. Tras tres primeras son comunes a la M_1 pero se incrementa el tiempo destinado al trabajo grupal, ya que tienen que realizar el trabajo correspondiente a la identificación y análisis del problema. Se incrementa también el tiempo del debate ya que se espera que las soluciones propuestas engloben a un mayor número de criterios (medioambiental, técnico, social, legislativos, salud, etc.). Se incorpora la metodología del trabajo autónomo, en la nueva fase 4, con el objetivo de favorecer la reflexión de los estudiantes en cuanto a la percepción de la adquisición de la competencia CT-10. Para esto se empleará la rúbrica de evaluación mostrada en la Tabla 4. Cabe indicar que esta rúbrica también se empleará por parte del profesorado para la evaluación de los estudiantes. Finalmente indicar que, mediante la encuesta de la actividad, se pretende recoger el "feed-back" directo del alumno sobre la actividad realizada.

Tabla 3. Nueva propuesta metodológica para la CT-10 (M_2)

\begin{tabular}{|c|c|c|c|}
\hline Fase actividad & $\begin{array}{l}\text { Metodología } \\
\text { docente } \\
\text { (duración) }\end{array}$ & $\begin{array}{l}\text { Recursos } \\
\text { didácticos }\end{array}$ & Tarea alumno \\
\hline $\begin{array}{l}\text { FASE 1: } \\
\text { Introducción tarea } \\
\text { - } \quad \text { Objetivo actividad } \\
\text { - } \quad \text { Exposición problema } \\
\text { - } \quad \text { Modo evaluación }\end{array}$ & $\begin{array}{l}\text { Lección } \\
\text { magistral } \\
(20 \mathrm{~min})\end{array}$ & Rúbrica & \\
\hline $\begin{array}{l}\text { FASE 2: } \\
\text { Propuesta de soluciones } \\
\text { - } \quad \text { Lectura documento } \\
\text { - } \quad \text { Elaboración } \\
\quad \text { propuestas } \\
\text { - } \quad \text { Contraste de opiniones } \\
\text { intergrupal }\end{array}$ & $\begin{array}{l}\text { Trabajo } \\
\text { grupal } \\
3-4 \text { personas } \\
(140 \mathrm{~min})\end{array}$ & $\begin{array}{l}\text { Fuentes } \\
\text { bibliográficas, } \\
\text { noticias, } \\
\text { documentales, } \\
\text { entrevistas, etc. } \\
\text { Cuestionario }\end{array}$ & $\begin{array}{l}\text { Elaboración informe } \\
\text { ( } 2 \text { hojas máximo) } \\
\text { Redactar propuesta soluciones } \\
\text { ( } 2 \text { hojas máximo) }\end{array}$ \\
\hline $\begin{array}{l}\text { FASE 3: } \\
\text { Debate } \\
\text { - } \quad \text { Exposición de } \\
\quad \text { propuestas } \\
\text { - } \quad \text { Debate pros/contra de } \\
\quad \text { soluciones }\end{array}$ & $\begin{array}{l}\text { Debate } \\
(60 \mathrm{~min})\end{array}$ & $\begin{array}{l}\text { Propuesta de } \\
\text { soluciones de la } \\
\text { FASE } 2\end{array}$ & $\begin{array}{l}\text { Cada grupo defiende las } \\
\text { soluciones planteadas. A través } \\
\text { del debate intentarán consensuar } \\
\text { una solución óptima. }\end{array}$ \\
\hline $\begin{array}{l}\text { FASE 4: } \\
\text { Autoevaluación/Evaluación } \\
\text { actividad }\end{array}$ & $\begin{array}{l}\text { Trabajo } \\
\text { autónomo } \\
\text { (20 } \mathrm{min})\end{array}$ & $\begin{array}{l}\text { Rúbrica } \\
\text { Encuesta }\end{array}$ & $\begin{array}{l}\text { Autoevaluación } \\
\text { Valorar actividad realizada } \\
\text { (encuesta) }\end{array}$ \\
\hline
\end{tabular}


Cambios metodológicos para la adquisición y evaluación de la competencia transversal "Conocimiento de los problemas contemporáneos"

\section{Resultados}

En este apartado se recoge la rúbrica de evaluación previa y la rúbrica modificada así como la encuesta para la evaluación de la actividad por parte del alumno.

La rúbrica de evaluación previa es la suministrada por el ICE de la UPV para nivel de dominio III (Fig. 1).

Fig. 1 Rúbrica UPV CT-10. Nivel de dominio III

\begin{tabular}{|c|c|c|c|c|c|}
\hline 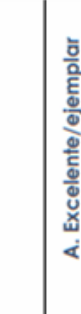 & 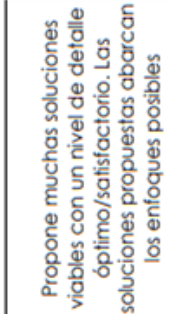 & 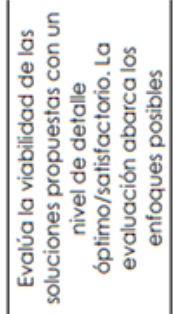 & 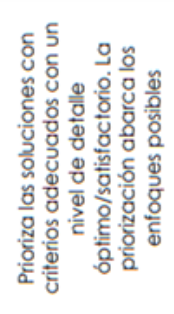 & 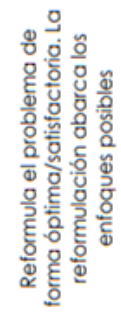 & 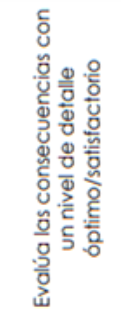 \\
\hline 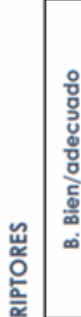 & 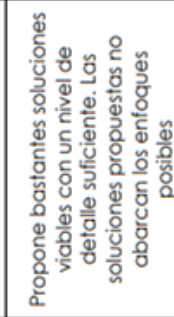 & 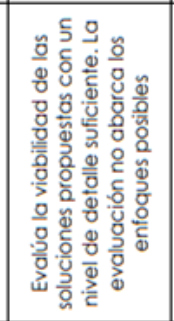 & 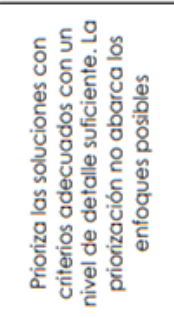 & 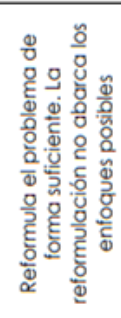 & 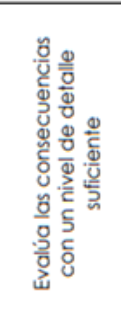 \\
\hline 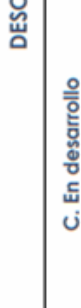 & 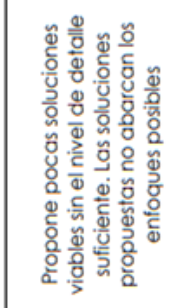 & 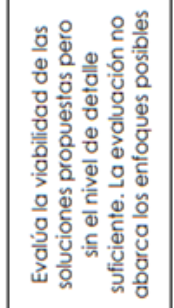 & 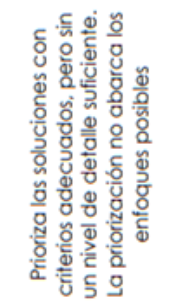 & 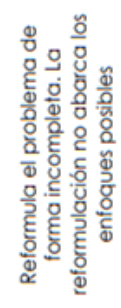 & 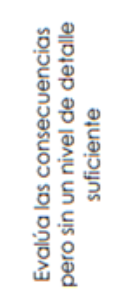 \\
\hline 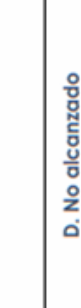 & 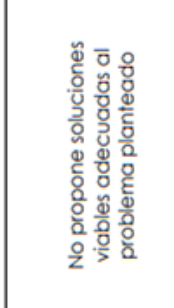 & 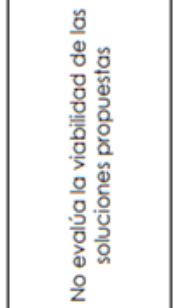 & 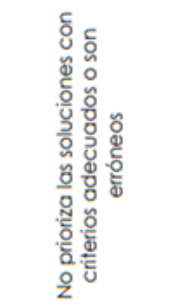 & 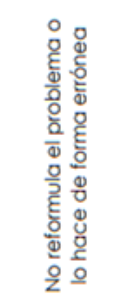 & 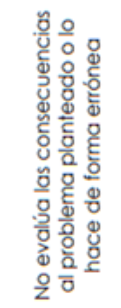 \\
\hline 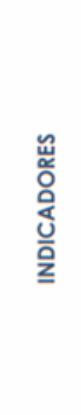 & 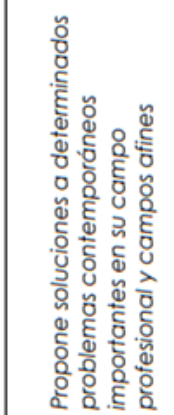 & 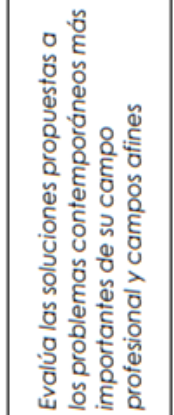 & 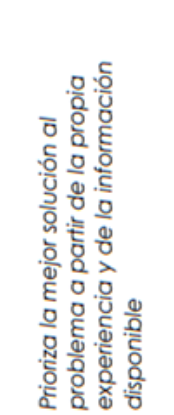 & 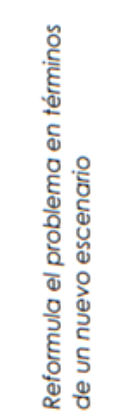 & 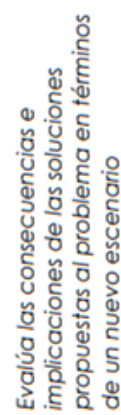 \\
\hline
\end{tabular}

(c) BY-NC-ND 2019, UniversitatPolitècnica de València 
En la Tabla 4 se muestra la rúbrica adaptada que incluye los tres dominios competenciales que se van a trabajar a través de la actividad.

Tabla 4. Rúbrica adaptada para evaluar la CT-10 nivel de dominio I, II y III.

\begin{tabular}{|c|c|c|c|c|}
\hline \multirow{2}{*}{ INDICADOR } & \multicolumn{4}{|c|}{ DESCRIPTORES } \\
\hline & D. No alcanzado & C. En desarrollo & B. Adecuado & A. Excelente \\
\hline $\begin{array}{l}\text { Identifica/Describe } \\
\text { el problema y sus } \\
\text { características }\end{array}$ & $\begin{array}{c}\text { No describe el } \\
\text { problema }\end{array}$ & $\begin{array}{c}\text { Describe } \\
\text { parcialmente el } \\
\text { problema }\end{array}$ & $\begin{array}{c}\text { Describe el } \\
\text { problema } \\
\text { considerando } \\
\text { casi todas sus } \\
\text { características }\end{array}$ & $\begin{array}{c}\text { Describe } \\
\text { perfectamente el } \\
\text { problema }\end{array}$ \\
\hline $\begin{array}{l}\text { Selecciona fuentes } \\
\text { diversas y } \\
\text { relevantes para la } \\
\text { búsqueda de } \\
\text { información desde } \\
\text { el punto de vista } \\
\text { relevancia, } \\
\text { fiabilidad, } \\
\text { actualidad }\end{array}$ & $\begin{array}{l}\text { Las fuentes de } \\
\text { información } \\
\text { consultadas y } \\
\text { seleccionadas no } \\
\text { son válidas para } \\
\text { desarrollar la } \\
\text { actividad }\end{array}$ & $\begin{array}{l}\text { Las fuentes de } \\
\text { información } \\
\text { consultadas y } \\
\text { seleccionadas } \\
\text { son insuficientes } \\
\text { para desarrollar } \\
\text { la actividad }\end{array}$ & $\begin{array}{l}\text { La mayoría de } \\
\text { las fuentes de } \\
\text { información } \\
\text { consultadas y } \\
\text { seleccionadas } \\
\text { son adecuadas } \\
\text { para desarrollar } \\
\text { la actividad }\end{array}$ & $\begin{array}{l}\text { Las fuentes de } \\
\text { información } \\
\text { consultadas y } \\
\text { seleccionadas } \\
\text { son adecuadas } \\
\text { para desarrollar } \\
\text { la actividad }\end{array}$ \\
\hline $\begin{array}{l}\text { Analiza las causas } \\
\text { del problema }\end{array}$ & $\begin{array}{c}\text { No se analizan } \\
\text { las causas del } \\
\text { problema }\end{array}$ & $\begin{array}{c}\text { Se analizan } \\
\text { correctamente } \\
\text { algunas de las } \\
\text { causas del } \\
\text { problema }\end{array}$ & $\begin{array}{c}\text { Se analizan } \\
\text { correctamente } \\
\text { casi todas las } \\
\text { causas del } \\
\text { problema }\end{array}$ & $\begin{array}{c}\text { Se analizan } \\
\text { correctamente } \\
\text { todas las causas } \\
\text { del problema }\end{array}$ \\
\hline $\begin{array}{l}\text { Analiza las } \\
\text { consecuencias del } \\
\text { problema }\end{array}$ & $\begin{array}{c}\text { No se analizan } \\
\text { las consecuencias } \\
\text { del problema }\end{array}$ & $\begin{array}{c}\text { Se analizan } \\
\text { correctamente } \\
\text { algunas de las } \\
\text { consecuencias } \\
\text { del problema }\end{array}$ & $\begin{array}{c}\text { Se analizan } \\
\text { correctamente } \\
\text { casi todas las } \\
\text { consecuencias } \\
\text { del problema }\end{array}$ & $\begin{array}{c}\text { Se analizan } \\
\text { correctamente } \\
\text { todas las } \\
\text { consecuencias } \\
\text { del problema }\end{array}$ \\
\hline $\begin{array}{l}\text { Propone y evalúa } \\
\text { soluciones al } \\
\text { problema teniendo } \\
\text { en cuenta los pros } \\
\text { y contra de cada } \\
\text { solución }\end{array}$ & $\begin{array}{l}\text { No propone ni } \\
\text { evalúa soluciones } \\
\text { al problema } \\
\text { teniendo en } \\
\text { cuenta los pros y } \\
\text { contra }\end{array}$ & $\begin{array}{c}\text { Propone } \\
\text { soluciones pero } \\
\text { no las evalúa } \\
\text { teniendo en } \\
\text { cuenta los pros y } \\
\text { contra }\end{array}$ & $\begin{array}{c}\text { Propone y evalúa } \\
\text { soluciones al } \\
\text { problema } \\
\text { teniendo en } \\
\text { cuenta algunos } \\
\text { pros y contra }\end{array}$ & $\begin{array}{c}\text { Propone y evalúa } \\
\text { soluciones al } \\
\text { problema } \\
\text { teniendo en } \\
\text { cuenta todos los } \\
\text { pros y contra }\end{array}$ \\
\hline
\end{tabular}

Para la realización de la encuesta se han preparado diez preguntas que serán contestadas según la escala likert (Totalmente de acuerdo/ Bastante de acuerdo /Indiferente /Bastante en desacuerdo /Totalmente en desacuerdo). La batería de preguntas se recoge en la Tabla 5. 
Cambios metodológicos para la adquisición y evaluación de la competencia transversal "Conocimiento de los problemas contemporáneos"

Tabla 5. Preguntas para la encuesta de evaluación de la actividad

\begin{tabular}{cl}
\hline $\mathbf{N}^{\mathbf{0}}$ Pregunta & Enunciado pregunta \\
\hline 1 & La actividad realizada me despierta interés. \\
2 & Las fases de la actividad son adecuadas para adquirir la competencia. \\
3 & La actividad realizada fomenta la adquisición de la competencia. \\
4 & La metodología empleada en la actividad será útil en tu futuro profesional para \\
trabajar \\
5 & El tiempo dedicado de 4 horas a la actividad es adecuado. \\
6 & Las actividades deben realizarse íntegramente en el aula \\
7 & Se ha trabajado de forma equitativa en el grupo. \\
8 & El debate favorece la actitud reflexiva y crítica \\
9 & La identificación y análisis del problema es clave para la búsqueda de soluciones \\
10 & La autoevaluación fomenta la reflexión sobre el trabajo realizado.
\end{tabular}

\section{Conclusiones}

- A partir de la experiencia obtenida durante tres años referente a la incorporación y evaluación de la adquisición de la competencia transversal "Conocimiento de los problemas contemporáneos", se detecta la falta de consolidación de los dominios competenciales I (identificación) y II (análisis).

- La metodología previa diseñada en esta la asignatura de máster, que no contemplaba el trabajo de los dominios I y II, y la dificultad de incorporar dicha competencia en los cursos del Grado justifica que el 75\% de los estudiantes de la asignatura tuvieran una adquisición de la competencia sólo aceptable.

- Los cambios en la metodología consisten en incluir la identificación y análisis del problema, permitiendo a los estudiantes seleccionar las fuentes de información que consideren más relevantes, para elaborar el informe y la propuesta de soluciones. Además se incrementan los tiempos de trabajo pero dentro del aula, y se incluye una etapa de autoevaluación y evaluación de la actividad realizada mediante la preparación de una encuesta.

- La rúbrica de evaluación y autoevaluación se ha modificado para incluir los tres niveles de dominio de la competencia $\mathrm{y}$, por tanto, las tres fases: IDENTIFICACIÓN, ANÁLISIS Y PROPUESTA SOLUCIONES, que incluye la evaluación de las soluciones propuestas. 


\section{Referencias}

BARRENETXEA AYESTA, M., MIJANGOS DEL CAMPO, J. J., GONZÁLEZ LASQUIBAR, X., BARANDIARAN GALDÓS, M., CARDONA RODRÍGUEZ, A. "Debates y juegos de rol, una vía para desarrollar competencias transversales en los grados de la Universidad del País Vasco/Euskal Herriko Unibertsitatea/UPV/EHU Grados de Experiencia de innovación aplicada en el grado de Gestión de Negocios". X Congreso Internacional de Docencia Universitaria e Innovación (CIDUI), (4-6 de julio, Palacio de Congresos de Girona, Barcelona, España) Girona, 2018. Revista CIDIU 2018, pp. 1-15

DE MIGUEL DÍAZ, M., ALFARO ROCHER, I.J., APODACA URQUIJO, P. et al. (2006) Modalidades de enseñanzas centradas en el desarrollo de competencias. Orientaciones para promover el cambio metodológico en el Espacio Europeo de Educación Superior. Oviedo: Ediciones Universidad de Oviedo.

FERNÁNDEZ-FERRER, M. ; FORÉS-MIRAVALLES, A. (2018). "Evaluación del desarrollo competencial en la educación superior. La perspectiva del profesorado universitario" en Educar. vol. 54/2, pp. 391-410

HERRERO, R., FERRER, M.A., CALDERÓN, A. (2014) "Evaluación de las competencias genéricas mediante rúbricas" 7 Competencias UPCT. Editorial: Universidad Politécnica de Cartagena, pp. 1323

MUDARRA PONS, C., TINTORÉ ESPUNY, M., BALAGUER FÀBREGAS, M.C. (2012) "Guía para evaluar competencias genéricas y específicas de titulación: Rúbricas en la Facultad de Educación". X Jornadas Redes de Investigación en Docencia Universitaria (7-8 junio 2012). UA, Alicante, 2012. https://web.ua.es/es/ice/jornadas-redes-2012/documentos/posters/245920.pdf

ORTEGA GUTIERREZ, J., ESTEBAN GARCÍA, L. (2017). "El debate como herramienta de aprendizaje". VIII Jornada de Innovación e Investigación Docente (15 Septiembre, Sevilla), Actas de las Jornadas conjuntas de innovación docente, investigación y transferencia, pp. 48-56.

PIQUÉ, B., FORÉS, A. (2012). Propuestas metodológicas para la educación superior. Barcelona: Universitat de Barcelona. Dipòsit Digital http://hdl.handle.net/2445/30702

QUIRÓS, C., RUBIO, M.J., TORRADO, M. (2018). “Àrees clau de la innovació docent a la Universitat de Barcelona. Línies d'innovació prioritàries als projectes d'innovació". X Congreso Internacional de Docencia Universitaria e Innovación (CIDUI)(4-6 de julio, Palacio de Congresos de Girona, Barcelona, España) Girona, 2018. Revista CIDIU 2018, pp. 1-12

UPV, 2018 http://www.upv.es/contenidos/

ZABALA, A., ARNAU, L. (2008) 11 Ideas clave. Cómo aprender y enseñar competencias. Barcelona: Editorial Graó 